\title{
A New Fixed Point Theorem on Generalized Quasimetric Spaces
}

\author{
Luljeta Kikina, ${ }^{1}$ Kristaq Kikina, $^{1}$ and Kristaq Gjino ${ }^{2}$ \\ ${ }^{1}$ Department of Mathematics and Computer Sciences, University of Gjirokastra, Gjirokastra, Albania \\ ${ }^{2}$ Department of Mathematics, University of Tirana, Tirana, Albania
}

Correspondence should be addressed to Luljeta Kikina, gjonileta@yahoo.com

Received 2 November 2011; Accepted 30 November 2011

Academic Editor: R. Avery

Copyright (C) 2012 Luljeta Kikina et al. This is an open access article distributed under the Creative Commons Attribution License, which permits unrestricted use, distribution, and reproduction in any medium, provided the original work is properly cited.

We obtain a new fixed point theorem in generalized quasimetric spaces. This result generalizes, unify, enrich, and extend some theorems of well-known authors from metric spaces to generalized quasimetric spaces.

\section{Introduction and Preliminaries}

The concept of metric space, as an ambient space in fixed point theory, has been generalized in several directions. Some of such generalizations are quasimetric spaces, generalized metric spaces, and generalized quasimetric spaces.

The concept of quasimetric spaces is treated differently by many authors. In this paper our concept is in line with this treated in [1-6], and so forth and the triangular inequality $d(x, y) \leq d(x, z)+d(z, y)$ is replaced by quasi-triangular inequality:

$$
d(x, y) \leq k[d(x, z)+d(z, y)], \quad k \geq 1 \text {. }
$$

In 2000 Branciari [7] introduced the concept of generalized metric spaces (gms) (the triangular inequality $d(x, y) \leq d(x, z)+d(z, y)$ is replaced by tetrahedral inequality $d(x, y) \leq$ $d(x, z)+d(z, w)+d(w, y))$. Starting with the paper of Branciari, some classical metric fixed point theorems have been transferred to gms (see [8-13]).

Recently L. kikina and k. kikina [14] introduced the concept of generalized quasimetric space (gqms) on the lines of quasimetric space, where the tetrahedral inequality $d(x, y) \leq$ $d(x, z)+d(z, w)+d(w, y)$ has been replaced by quasitetrahedral inequality $d(x, y) \leq$ $k[d(x, z)+d(z, w)+d(w, y)]$. The well-known fixed point theorems of Banach and of Kannan have been transferred to such a space. 
The metric spaces are a special case of generalized metric spaces and generalized metric spaces are a special case of generalized quasimetric spaces (for $k=1$ ). Also, every qms is a gqms, while the converse is not true.

In gqms, contrary to a metric space, the "open" balls $B(a, r)=\{x \in X: d(x, a)<r\}$ are not always open sets, and consequently, a generalized quasidistance is not always continuous of its variables. The gqms is not always a Hausdorff space and the convergent sequence $\left(x_{n}\right)$ in gqms is not always a Cauchy sequence (see Example 1.3).

Under this situation, it is reasonable to consider if some well-known fixed point theorems can be obtained in generalized quasimetric space.

The aim of this paper is to generalize, unify, and extend some theorems of well-known authors such as of Fisher and Popa, from metric spaces to generalized quasimetric spaces.

Let us start with the main definitions.

Definition 1.1 (see [7]). Let $X$ be a set and $d: X^{2} \rightarrow R^{+}$a mapping such that for all $x, y \in X$ and for all distinct points $z, w \in X$, each of them different from $x$ and $y$, one has

(a) $d(x, y)=0$ if and only if $x=y$,

(b) $d(x, y)=d(y, x)$,

(c) $d(x, y) \leq d(x, z)+d(z, w)+d(w, y)$ (tetrahedral inequality).

Then $d$ is called a generalized metric and $(X, d)$ is a generalized metric space (or shortly gms).

Definition 1.2 (see [14]). Let $X$ be a set. A nonnegative symmetric function $d$ defined on $X \cdot X$ is called a generalized quasidistance on $X$ if and only if there exists a constant $k \geq 1$ such that for all $x, y \in X$ and for all distinct points $z, w \in X$, each of them different from $x$ and $y$, the following conditions hold:

(i) $d(x, y)=0 \Leftrightarrow x=y$;

(ii) $d(x, y)=d(y, x)$;

(iii) $d(x, y) \leq k[d(x, z)+d(z, w)+d(w, y)]$.

Inequality (2.7) is often called quasitetrahedral inequality and $k$ is often called the coefficient of $d$. A pair $(X, d)$ is called a generalized quasimetric space if $X$ is a set and $d$ is a generalized quasidistance on $X$.

The set $B(a, r)=\{x \in X: d(x, a)<r\}$ is called "open" ball with center $a \in X$ and radius $r>0$.

The family $\tau=\{Q \subset X: \forall a \in Q, \exists r>0, B(a, r) \subset Q\}$ is a topology on $X$ and it is called induced topology by the generalized quasidistance $d$.

The following example illustrates the existence of the generalized quasimetric space for an arbitrary constant $k \geq 1$. 
Example 1.3 (see [14]). Let $X=\{1-(1 / n): n=1,2, \ldots\} \cup\{1,2\}$. Define $d: X \cdot X \rightarrow R$ as follows:

$$
d(x, y)= \begin{cases}0, & \text { for } x=y \\ \frac{1}{n}, & \text { for } x \in\{1,2\}, \quad y=1-\frac{1}{n} \text { or } y \in\{1,2\}, x=1-\frac{1}{n}, x \neq y \\ 3 k, & \text { for } x, y \in\{1,2\}, \quad x \neq y \\ 1, & \text { otherwise. }\end{cases}
$$

Then it is easy to see that $(X, d)$ is a generalized quasimetric space and is not a generalized metric space (for $k>1$ ).

Note that the sequence $\left(x_{n}\right)=(1-(1 / n))$ converges to both 1 and 2 and it is not a Cauchy sequence:

$$
d\left(x_{n}, x_{m}\right)=d\left(1-\frac{1}{n}, 1-\frac{1}{m}\right)=1, \quad \forall n, m \in N
$$

Since $B(1, r) \cap B(2, r) \neq \phi$ for all $r>0$, the $(X, d)$ is non-Hausdorff generalized metric space.

The function $d$ is not continuous: $1=\lim _{n \rightarrow \infty} d(1-(1 / n), 1 / 2) \neq d(1,1 / 2)=1 / 2$.

In [14] the following is proved.

Proposition 1.4. If $(X, d)$ is a quasimetric space, then $(X, d)$ is a generalized quasimetric space. The converse proposition does not hold true.

Definition 1.5. A sequence $\left\{x_{n}\right\}$ in a generalized quasimetric space $(X, d)$ is called Cauchy sequence if $\lim _{n, m \rightarrow \infty} d\left(x_{n}, x_{m}\right)=0$.

Definition 1.6. Let $(X, d)$ be a generalized quasimetric space. Then one has the following.

(1) A sequence $\left\{x_{n}\right\}$ in $X$ is said to be convergent to a point $x \in X$ (denoted by $\left.\lim _{n \rightarrow \infty} x_{n}=x\right)$ if $\lim _{n \rightarrow \infty} d\left(x_{n}, x\right)=0$.

(2) It is called compact if every sequence contains a convergent subsequence.

Definition 1.7. A generalized quasimetric space $(X, d)$ is called complete, if every Cauchy sequence is convergent.

Definition 1.8. Let $(X, d)$ be a gqms and the coefficient of $d$ is $k$.

A map $T: X \rightarrow X$ is called contraction if there exists $0<c<1 / k$ such that

$$
d(T x, T y) \leq c d(x, y) \quad \forall x, y \in X
$$

Definition 1.9. Let $T: X \rightarrow X$ be a mapping where $X$ is a gqms. For each $x \in X$, let

$$
O(x)=\left\{x, T x, T^{2} x, \ldots\right\}
$$


which will be called the orbit of $T$ at $x$. The space $X$ is said to be $T$-orbitally complete if and only if every Cauchy sequence which is contained in $O(x)$ converges to a point in $X$.

Definition 1.10. The set of all upper semicontinuous functions with 3 variables $f: R_{+}^{3} \rightarrow R$ satisfying the following properties:

(a) $f$ is nondecreasing in respect to each variable,

(b) $f(t, t, t) \leq t, t \in R_{+}$

will be noted by $\mathbb{F}_{3}$ and every such function will be called an $\mathbb{F}_{3}$-function. Some examples of $\mathbb{F}_{3}$-function are as follows:

(1) $f\left(t_{1}, t_{2}, t_{3}\right)=\max \left\{t_{1}, t_{2}, t_{3}\right\}$,

(2) $f\left(t_{1}, t_{2}, t_{3}\right)=\left[\max \left\{t_{1} t_{2}, t_{2} t_{3}, t_{3} t_{1}\right\}\right]^{1 / 2}$,

(3) $f\left(t_{1}, t_{2}, t_{3}\right)=\left[\max \left\{t_{1}^{p}, t_{2}^{p}, t_{3}^{p}\right\}\right]^{1 / p}, p>0$,

(4) $f\left(t_{1}, t_{2}, t_{3}\right)=\left(a t_{1} t_{2}+b t_{2} t_{3}+c t_{3} t_{1}\right)^{1 / 2}$, where $a, b, c \geq 0$ and $a+b+c<1$.

\section{Main Result}

We state the following lemma which we will use for the proof of the main theorem.

Lemma 2.1. Let $(X, d)$ be a generalized quasimetric space and $\left\{x_{n}\right\}$ is a sequence of distinct point $\left(x_{n} \neq x_{m}\right.$ for all $\left.n \neq m\right)$ in X. If $d\left(x_{n}, x_{n+1}\right) \leq c^{n} l, 0 \leq c<1 / k<1$, for all $n \in N$ and $\lim _{n \rightarrow \infty} d\left(x_{n}, x_{n+2}\right)=0$, then $\left\{x_{n}\right\}$ is a Cauchy sequence.

Proof. If $m>2$ is odd, then writing $m=2 p+1, p \geq 1$, by quasitetrahedral inequality, we can easily show that

$$
\begin{aligned}
d\left(x_{n}, x_{n+m}\right) & \leq k\left[d\left(x_{n}, x_{n+1}\right)+d\left(x_{n+1}, x_{n+2}\right)+d\left(x_{n+2}, x_{n+m}\right)\right] \\
& \leq k d\left(x_{n}, x_{n+1}\right)+k^{2} d\left(x_{n+1}, x_{n+2}\right)+k^{2} d\left(x_{n+2}, x_{n+m}\right) \\
& \leq k c^{n} l+k^{2} c^{n+1} l+k^{2} d\left(x_{n+2}, x_{n+m}\right) \leq \cdots \\
& \leq k c^{n} l+k^{2} c^{n+1} l+k^{3} c^{n+2} l+\cdots+k^{m-1} c^{n+m-2} l+k^{m-1} c^{n+m-1} l \\
& \leq k c^{n} l+k^{2} c^{n+1} l+k^{3} c^{n+2} l+\cdots+k^{m-1} c^{n+m-2} l+k^{m} c^{n+m-1} l \\
& \leq k c^{n} l\left[1+k c+\cdots+(k c)^{m-1}\right]=k c^{n} l \frac{1-(k c)^{m}}{1-k c}<k c^{n} l \frac{1}{1-k c} .
\end{aligned}
$$

Therefore, $\lim _{n \rightarrow \infty} d\left(x_{n}, x_{n+m}\right)=0$. 
If $m>2$ is even, then writing $m=2 p, p \geq 2$ and using the same arguments as before we can get

$$
\begin{aligned}
d\left(x_{n}, x_{n+m}\right) & \leq k\left[d\left(x_{n}, x_{n+2}\right)+d\left(x_{n+2}, x_{n+3}\right)+d\left(x_{n+3}, x_{n+m}\right)\right] \\
& \leq k d\left(x_{n}, x_{n+2}\right)+k c^{n+2} l+k d\left(x_{n+3}, x_{n+m}\right) \\
& \leq k d\left(x_{n}, x_{n+2}\right)+k c^{n+2} l+k^{2}\left[d\left(x_{n+3}, x_{n+4}\right)+d\left(x_{n+4}, x_{n+5}\right)+d\left(x_{n+5}, x_{n+m}\right)\right] \leq \cdots \\
& \leq k d\left(x_{n}, x_{n+2}\right)+k c^{n+2} l+k^{2} c^{n+3} l+\cdots+k^{m-2} c^{n+m-1} l \\
& =k d\left(x_{n}, x_{n+2}\right)+k c^{n+2} l\left[1+k c+\cdots+(k c)^{m-3}\right] \\
& =k d\left(x_{n}, x_{n+2}\right)+k c^{n+2} l \frac{1-(k c)^{m-2}}{1-k c} \\
& <k d\left(x_{n}, x_{n+2}\right)+k c^{n+2} l \frac{1}{1-k c}
\end{aligned}
$$

And so $\lim _{n \rightarrow \infty} d\left(x_{n}, x_{n+m}\right)=0$. It implies that $\left\{x_{n}\right\}$ is a Cauchy sequence in $X$. This completes the proof of the lemma.

We state the following theorem.

Theorem 2.2. Let $(X, d)$ and $(Y, \rho)$ be two generalized quasimetric spaces with coefficients $k_{1}$ and $k_{2}$, respectively. Let $T$ be a mapping of $X$ into $Y$ and $S$ a mapping of $Y$ into $X$ satisfying the following inequalities:

$$
\begin{aligned}
& d(S y, S T x) \leq c f_{1}\{d(x, S y), d(x, S T x), \rho(y, T x)\}, \\
& \rho(T x, T S y) \leq c f_{2}\{\rho(y, T x), \rho(y, T S y), d(x, S y)\},
\end{aligned}
$$

for all $x \in X$ and $y \in Y$, where $0<c<1 / k \leq 1, k=\max \left\{k_{1}, k_{2}\right\}, f_{1}, f_{2} \in \mathbb{F}_{3}$. If there exists $x_{0} \in X$ such that $O\left(x_{0}\right)$ is ST-orbitally complete in $X$ and $O\left(T x_{0}\right)$ is TS-orbitally complete in $Y$, then ST has a unique fixed point $\alpha$ in $X$ and TS has a unique fixed point $\beta$ in $Y$. Further, T $\alpha=\beta$ and $S \beta=\alpha$.

Proof. Let $x_{0}$ be an arbitrary point in $X$. Define the sequences $\left(x_{n}\right)$ and $\left(y_{n}\right)$ inductively as follows:

$$
x_{n}=S y_{n}=(S T)^{n} x_{0}, \quad y_{1}=T x_{0}, \quad y_{n+1}=T x_{n}=(T S)^{n} y_{1}, \quad n \geq 1
$$

\section{Denote}

$$
d_{n}=d\left(x_{n}, x_{n+1}\right), \quad \rho_{n}=\rho\left(y_{n}, y_{n+1}\right), \quad n=1,2, \ldots
$$


Using the inequality (2) we get

$$
\begin{aligned}
\rho_{n} & =\rho\left(y_{n}, y_{n+1}\right)=\rho\left(T x_{n-1}, T S y_{n}\right) \\
& \leq c f_{2}\left(\rho\left(y_{n}, y_{n}\right), \rho\left(y_{n}, y_{n+1}\right), d\left(x_{n-1}, x_{n}\right)\right)=c f_{2}\left(0, \rho_{n}, d_{n-1}\right)
\end{aligned}
$$

By this inequality and properties of $f_{2}$, it follows that

$$
\rho_{n} \leq c d_{n-1}
$$

Using the inequality (2.3) we have

$$
\begin{aligned}
d_{n} & =d\left(x_{n}, x_{n+1}\right)=d\left(S y_{n}, S T x_{n}\right) \\
& \leq c f_{1}\left(d\left(x_{n}, x_{n}\right), d\left(x_{n}, x_{n+1}\right), \rho\left(y_{n}, y_{n+1}\right)\right)=c f_{1}\left(0, d_{n}, \rho_{n}\right),
\end{aligned}
$$

and so $d_{n} \leq c \rho_{n}$. By this inequality and (2.7) we obtain

$$
d_{n} \leq c^{2} d_{n-1} \leq c d_{n-1}
$$

Using the mathematical induction, by the inequalities (2.7) and (2.9), we get

$$
d_{n} \leq c^{n} d\left(x_{0}, x_{1}\right), \quad \rho_{n} \leq c^{n} d\left(x_{0}, x_{1}\right)
$$

So

$$
\lim _{n \rightarrow \infty} d\left(x_{n}, x_{n+1}\right)=\lim _{n \rightarrow \infty} \rho\left(y_{n}, y_{n+1}\right)=0 .
$$

Applying the inequality (2), we get

$$
\begin{aligned}
\rho\left(y_{n}, y_{n+2}\right) & =\rho\left(\operatorname{Tx}_{n-1}, T S y_{n+1}\right) \\
& \leq c f_{2}\left(\rho\left(y_{n+1}, y_{n}\right), \rho\left(y_{n+1}, y_{n+2}\right), d\left(x_{n-1}, x_{n+1}\right)\right) \\
& =c f_{2}\left(\rho_{n}, \rho_{n+1}, d\left(x_{n-1}, x_{n+1}\right)\right) \leq c \max \left\{c^{n} d\left(x_{0}, x_{1}\right), d\left(x_{n-1}, x_{n+1}\right)\right\}
\end{aligned}
$$

and so

$$
\rho\left(y_{n}, y_{n+2}\right) \leq \max \left\{c^{n} d\left(x_{0}, x_{1}\right), c d\left(x_{n-1}, x_{n+1}\right)\right\}
$$

Similarly, using (2.3), we obtain

$$
d\left(x_{n}, x_{n+2}\right) \leq \max \left\{c^{n} d\left(x_{0}, x_{1}\right), c d\left(x_{n-1}, x_{n+1}\right)\right\} .
$$


Using the mathematical induction, we get

$$
\begin{aligned}
d\left(x_{n}, x_{n+2}\right) & \leq \max \left\{c^{n} d\left(x_{0}, x_{1}\right), c d\left(x_{n-1}, x_{n+1}\right)\right\} \leq \max \left\{c^{n} d\left(x_{0}, x_{1}\right), c^{2} d\left(x_{n-2}, x_{n}\right)\right\} \\
& \leq \cdots \leq \max \left\{c^{n} d\left(x_{0}, x_{1}\right), c^{n} d\left(x_{0}, x_{2}\right)\right\}=c^{n} \max \left\{d\left(x_{0}, x_{1}\right), d\left(x_{0}, x_{2}\right)\right\}=c^{n} l,
\end{aligned}
$$

and so

$$
d\left(x_{n}, x_{n+2}\right) \leq c^{n} l, \quad \text { similarly } \rho\left(y_{n}, y_{n+2}\right) \leq c^{n} l,
$$

where $l=\max \left\{d\left(x_{0}, x_{1}\right), d\left(x_{0}, x_{2}\right)\right\}$.

We divide the proof into two cases.

Case 1. Suppose $x_{p}=x_{q}$ for some $p, q \in N, p \neq q$. Let $p>q$. Then $(S T)^{p} x_{0}=(S T)^{p-q}(S T)^{q} x_{0}=$ $(S T)^{q} x_{0}$; that is, $(S T)^{n} \alpha=\alpha$ where $n=p-q$ and $(S T)^{q} x_{0}=\alpha$. Now if $n>1$, by (2.10), we have

$$
d(\alpha, S T \alpha)=d\left[(S T)^{n} \alpha,(S T)^{n+1} \alpha\right] \leq c^{n} d(\alpha, S T \alpha)
$$

Since $0<c<1, d(\alpha, S T \alpha)=0$. So $S T \alpha=\alpha$ and hence $\alpha$ is a fixed point of $S T$.

By the equality $x_{p}=x_{q}$ it follows that $y_{p+1}=y_{q+1}$. We take $\beta=(T S)^{q} T x_{0}$ and, in similar way, we prove that $\beta$ is a fixed point of TS.

Case 2. Assume that $x_{n} \neq x_{m}$ for all $n \neq m$. Then, from (2.10), (2.16), and Lemma 2.1 is derived that $\left\{x_{n}\right\}$ is a Cauchy sequence in $X$. Since $O\left(x_{0}\right)$ is ST-orbitally complete, there exists $\alpha \in X$ such that $\lim _{n \rightarrow \infty} x_{n}=\alpha$. In the same way, we show that the sequence $\left(y_{n}\right)$ is a Cauchy sequence and there exists a $\beta \in Y$ such that $\lim _{n \rightarrow \infty} y_{n}=\beta$.

We now prove that the limits $\alpha$ and $\beta$ are unique. Suppose, to the contrary, that $\alpha^{\prime} \neq \alpha$ is also $\lim _{n \rightarrow \infty} x_{n}$. Since $x_{n} \neq x_{m}$ for all $n \neq m$, there exists a subsequence $\left(x_{n_{k}}\right)$ of $\left(x_{n}\right)$ such that $x_{n_{k}} \neq \alpha$ and $x_{n_{k}} \neq \alpha^{\prime}$ for all $k \in N$. Without loss of generality, assume that $\left(x_{n}\right)$ is this subsequence. Then by Tetrahedral property of Definition 1.1 we obtain

$$
d\left(\alpha, \alpha^{\prime}\right) \leq k\left[d\left(\alpha, x_{n}\right)+d\left(x_{n}, x_{n+1}\right)+d\left(x_{n+1}, \alpha^{\prime}\right)\right] .
$$

Letting $n$ tend to infinity we get $d\left(\alpha, \alpha^{\prime}\right)=0$ and so $\alpha=\alpha^{\prime}$, in the same way for $\beta$.

Let us prove now that $\alpha$ is a fixed point of $S T$. First we prove that $\beta=T \alpha$. In contrary, if $\beta \neq T \alpha$, the sequence $\left(y_{n}\right)$ does not converge to $T \alpha$ and there exists a subsequence $\left(y_{n_{q}}\right)$ of $\left(y_{n}\right)$ such that $y_{n_{q}} \neq T \alpha$ for all $q \in N$. Then by Tetrahedral property of Definition 1.1 we obtain

$$
\rho(\beta, T \alpha) \leq k\left[\rho\left(\beta, y_{n_{q-1}}\right)+\rho\left(y_{n_{q-1}}, y_{n_{q}}\right)+\rho\left(y_{n_{q}}, T \alpha\right)\right]
$$

Then if $q \rightarrow \infty$, we get

$$
\rho(\beta, T \alpha) \leq k \lim _{q \rightarrow \infty} \rho\left(y_{n_{q}}, T \alpha\right)
$$


Using the inequality (2), for $x=\alpha$ and $y=y_{n-1}$ we obtain

$$
\begin{aligned}
\rho\left(T \alpha, y_{n}\right)=\rho\left(T \alpha, T S y_{n-1}\right) & \leq c f_{2}\left(\rho\left(y_{n-1}, T \alpha\right), \rho\left(y_{n-1}, T S y_{n-1}\right), d\left(\alpha, S y_{n-1}\right)\right) \\
& =c f_{2}\left(\rho\left(y_{n-1}, T \alpha\right), \rho\left(y_{n-1}, y_{n}\right), d\left(\alpha, x_{n-1}\right)\right) .
\end{aligned}
$$

Letting $n$ tend to infinity we get

$$
\lim _{n \rightarrow \infty} \rho\left(T \alpha, y_{n}\right) \leq c f_{2}\left(\lim _{n \rightarrow \infty} \rho\left(y_{n-1}, T \alpha\right), 0,0\right)
$$

And so,

$$
\varlimsup_{n \rightarrow \infty} \rho\left(T \alpha, y_{n}\right)=0
$$

Since $\lim _{q \rightarrow \infty}^{--} \rho\left(y_{n_{q}}, T \alpha\right) \leq \lim _{n \rightarrow \infty}^{--} \rho\left(T \alpha, y_{n}\right)$, by (2.23) and (2.20), we have $\rho(\beta, T \alpha)=0$ and so

$$
T \alpha=\beta
$$

It follows similarly that

$$
S \beta=\alpha
$$

By (2.24) and (2.25) we obtain

$$
S T \alpha=S \beta=\alpha, \quad T S \beta=T \alpha=\beta .
$$

Thus, we proved that the points $\alpha$ and $\beta$ are fixed points of ST and TS, respectively.

Let us prove now the uniqueness (for Cases 1 and 2 in the same time). Assume that $\alpha^{\prime} \neq \alpha$ is also a fixed point of ST. By (2.3) for $x=\alpha^{\prime}$ and $y=\beta$ we get

$$
d\left(\alpha, \alpha^{\prime}\right)=d\left(S \beta, S T \alpha^{\prime}\right) \leq c f_{1}\left(d\left(\alpha^{\prime}, \alpha\right), 0, \rho\left(T \alpha, T \alpha^{\prime}\right)\right) .
$$

And so,we have

$$
d\left(\alpha, \alpha^{\prime}\right) \leq c \rho\left(T \alpha, T \alpha^{\prime}\right)
$$

If $T \alpha \neq T \alpha^{\prime}$, in similar way by (2) for $x=S T \alpha$ and $y=T \alpha^{\prime}$, we have

$$
\rho\left(T \alpha, T \alpha^{\prime}\right) \leq c d\left(\alpha, \alpha^{\prime}\right)
$$

By (2.28) and (2.29) we get $d\left(\alpha, \alpha^{\prime}\right)=0$. Thus, we have again $\alpha=\alpha^{\prime}$. The uniqueness of $\beta$ follows similarly. This completes the proof of the theorem. 


\section{Corollaries}

(1) If $k_{1}=k_{2}=1$, then by Theorem 2.2 we obtain [12, Theorem 2.1], that generalize and extend the well-known Fisher fixed point theorem [15] from metric space to generalized metric spaces.

For different expressions of $f_{1}$ and $f_{2}$ in Theorem 2.2 we get different theorems.

(2) For $f_{1}=f_{2}=f$, where $f\left(t_{1}, t_{2}, t_{3}\right)=\max \left\{t_{1}, t_{2}, t_{3}\right\}$ we have an extension of Fisher's theorem [15] in generalized quasimetric spaces.

(3) For $f_{1}=f_{2}=f$, where $f\left(t_{1}, t_{2}, t_{3}\right)=\left[\max \left\{t_{1} t_{2}, t_{2} t_{3}, t_{3} t_{1}\right\}\right]^{1 / 2}$, we have an extension of Popa's theorem [13] in generalized quasimetric spaces.

(4) For $f_{1}\left(t_{1}, t_{2}, t_{3}\right)=\left(a_{1} t_{1} t_{2}+b_{1} t_{2} t_{3}+c_{1} t_{3} t_{1}\right)^{1 / 2}$ and $f_{2}\left(t_{1}, t_{2}, t_{3}\right)=\left(a_{2} t_{1} t_{2}+b_{2} t_{2} t_{3}+c_{2} t_{3} t_{1}\right)^{1 / 2}$ we obtain an extension of Popa's Corollary [13] in generalized quasimetric spaces.

Remark 3.1. We can obtain many other similar results for different $f$.

\section{References}

[1] M. Bramanti and L. Brandolini, "Schauder estimates for parabolic nondivergence operators of Hörmander type," Journal of Differential Equations, vol. 234, no. 1, pp. 177-245, 2007.

[2] L. Kikina and K. Kikina, "A related fixed point theorem for $\mathrm{m}$ mappings on $\mathrm{m}$ complete quasi-metric spaces," Mathematica Cluj. In press.

[3] L. Kikina and K. Kikina, "Generalized fixed point theorem for three mappings on three quasi-metric spaces," Journal of Computational Analysis and Applications. In press.

[4] B. Pepo, "Fixed points for contractive mappings of third order in pseudo-quasimetric spaces," Indagationes Mathematicae, vol. 1, no. 4, pp. 473-481, 1990.

[5] C. Peppo, "Fixed point theorems for $(\phi, k, i, j)$-mappings," Nonlinear Analysis, vol. 72, no. 2, pp. 562-570, 2010.

[6] Q. Xia, "The geodesic problem in quasimetric spaces," Journal of Geometric Analysis, vol. 19, no. 2, pp. 452-479, 2009.

[7] A. Branciari, "A fixed point theorem of Banach-Caccioppoli type on a class of generalized metric spaces," Publicationes Mathematicae Debrecen, vol. 57, no. 1-2, pp. 31-37, 2000.

[8] A. Azam and M. Arshad, "Kannan fixed point theorem on generalized metric spaces," Journal of Nonlinear Sciences and Its Applications, vol. 1, no. 1, pp. 45-48, 2008.

[9] P. Das, "A fixed point theorem on a class of generalized metric spaces," Korean Journal of Mathematical Sciences, vol. 1, pp. 29-33, 2002.

[10] P. Das and L. K. Dey, "A fixed point theorem in a generalized metric space," Soochow Journal of Mathematics, vol. 33, no. 1, pp. 33-39, 2007.

[11] L. Kikina and K. Kikina, "A fixed point theorem in generalizedmetric spaces," Demonstratio Mathematica. In press.

[12] L. Kikina and K. Kikina, "Fixed points on two generalized metric spaces," International Journal of Mathematical Analysis, vol. 5, no. 29-32, pp. 1459-1467, 2011.

[13] I. R. Sarma, J. M. Rao, and S. S. Rao, “Contractions over generalized metric spaces,” Journal of Nonlinear Science and its Applications, vol. 2, no. 3, pp. 180-182, 2009.

[14] L. Kikina and K. Kikina, "Two fixed point theorems on a class of generalized quasi-metric spaces," Journal of Computational Analysis and Applications. In press.

[15] B. Fisher, "Fixed points on two metric spaces," Glasnik Matematički, vol. 16, no. 36, pp. 333-337, 1981. 


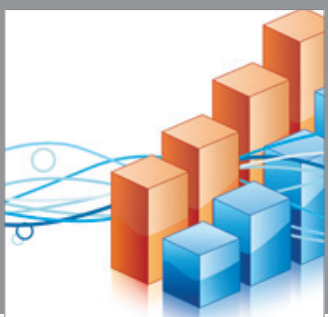

Advances in

Operations Research

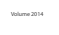

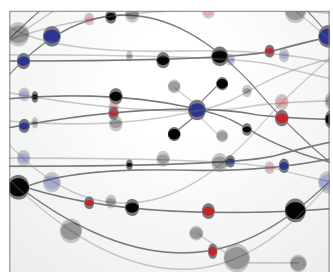

\section{The Scientific} World Journal
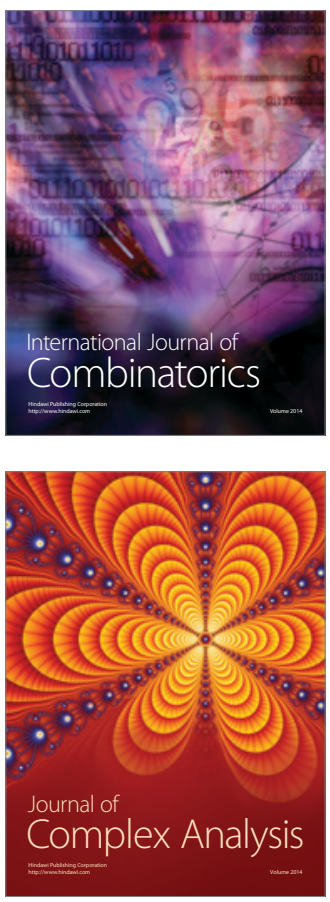

International Journal of

Mathematics and

Mathematical

Sciences
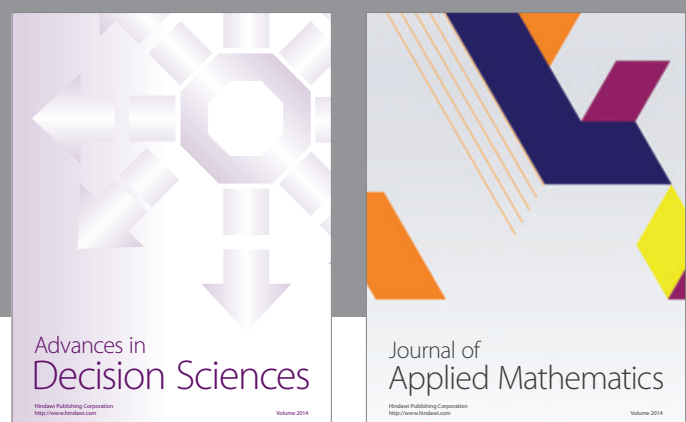

Journal of

Applied Mathematics
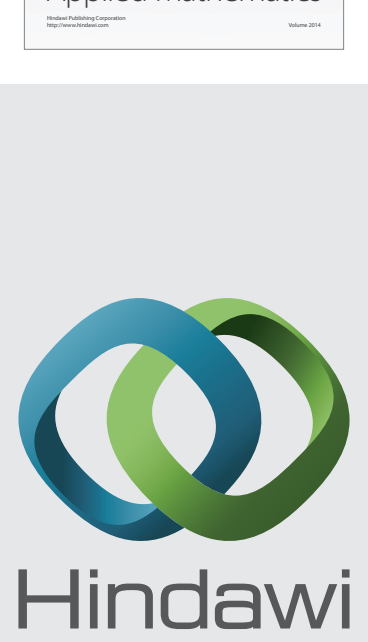

Submit your manuscripts at http://www.hindawi.com
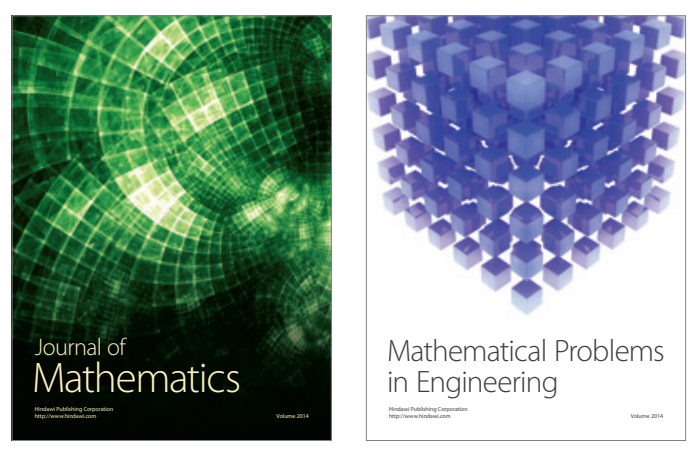

Mathematical Problems in Engineering
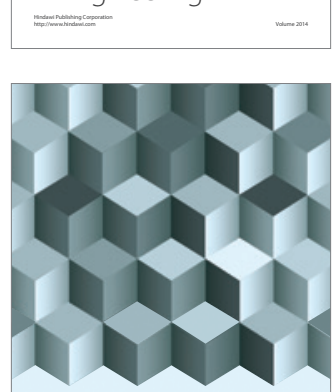

Journal of

Function Spaces
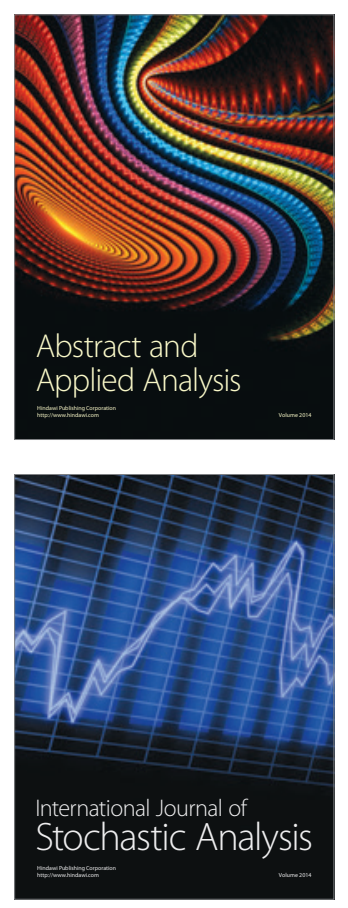

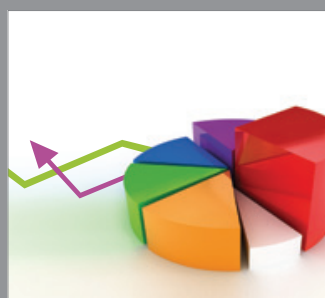

ournal of

Probability and Statistics

Promensencen
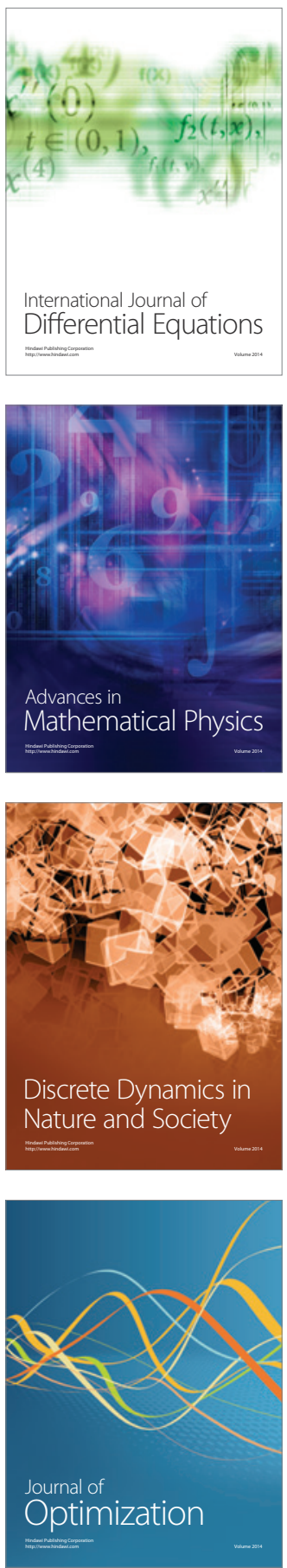